\title{
Comparison of Ambisense M RNA of Watermelon Silver Mottle Virus with Other Tospoviruses
}

\author{
Fang-Hua Chu and Shyi-Dong Yeh
}

Department of Plant Pathology, National Chung Hsing University, Taichung, Taiwan, Republic of China. Accepted for publication 19 December 1997.

\section{ABSTRACT}

Chu, F.-H., and Yeh, S.-D. 1998. Comparison of ambisense M RNA of watermelon silver mottle virus with other tospoviruses. Phytopathology 88:351-358.

Double-stranded genomic RNAs (dsRNAs) extracted from Chenopodium quinoa infected with watermelon silver mottle virus (WSMV) were similar to those of tomato spotted wilt virus (TSWV, serogroup I) and impatiens necrotic spot virus (INSV, serogroup III), except that the S dsRNA of WSMV is 0.75 and $0.6 \mathrm{kbp}$ longer than those of TSWV and INSV, respectively. The complete nucleotide sequence of the genomic M RNA of WSMV was determined from cDNA clones generated from separated M dsRNA. The M RNA is 4,880 nucleotides in length with two open reading frames (ORFs) in an ambisense organization. The M RNAencoded nonstructural (NSm) ORF located on the viral strand encodes a protein of 312 amino acids ( $35 \mathrm{kDa}$ ), and the G1/G2 ORF located on the viral complementary strand encodes a protein of 1,121 amino acids (127.6 $\mathrm{kDa}$ ). The RNA probe corresponding to the NSm or G1/G2 ORF of WSMV failed to hybridize with the M dsRNAs of TSWV and INSV. Comparison of M and S RNAs of WSMV, TSWV, INSV, and peanut bud necrosis virus (PBNV, serogroup IV) revealed a consensus sequence of eight nucleotides of $5^{\prime}$-AGAGCAAU...-3' at their $5^{\prime}$ ends and $5^{\prime}$-...AUUGCUCU-3' at their $3^{\prime}$ ends. The low overall nucleotide identities (56.4 to 56.9\%) of the M RNA and the low amino acid identities of the NSm and G1/G2 proteins (30.5 to 40.9\%) with those of TSWV and INSV indicate that WSMV belongs to the Tospovirus genus but is phylogenetically distinct from viruses in serogroups I and III. The M RNA of WSMV shares a nucleotide identity of $79.6 \%$ with that of PBNV, and the two viruses share 83.4 and $88.7 \%$ amino acid identities for their NSm and G1/G2 proteins, respectively. It is concluded that they are two related but distinct species of serogroup IV. In addition to the viral or viral complementary full-length M RNA, two putative RNA messages for the NSm gene and the G1/G2 gene, 1.0 and $3.4 \mathrm{~kb}$, respectively, were detected from the total RNA extracted from WSMV-infected tissue of Nicotiana benthamiana. The 1.0- and 3.4-kb RNAs were also detected in the viral RNAs extracted from purified nucleocapsids, suggesting that the putative messages of the M RNA of WSMV can also be encapsidated by the nucleocapsid protein.
The genus Tospovirus within the family Bunyaviridae encompasses a group of enveloped plant viruses that cause great losses in many economically important crops worldwide (10). The genus is characterized by quasi-spherical, enveloped particles containing three single-stranded RNA (ssRNA) segments denoted S RNA, M RNA, and L RNA $(27,40)$. The L RNA is of negative polarity and encodes a putative RNA polymerase that may be associated with the ribonucleoprotein complex $(5,19)$. The other two genomic RNAs use an ambisense coding strategy. The M RNA encodes a nonstructural $(\mathrm{NSm})$ protein in the viral (v) sense and a protein as the precursor for the G1 and G2 glycoproteins in the viral complementary (vc) sense $(18,25)$. The NSm protein may be involved in cell-to-cell movement of nonenveloped ribonucleocapsid structures (21). Tubular structures are specifically induced upon expression of the NSm gene in both plant and insect cell systems (38). The G1 and G2 glycoproteins are considered to form spikes on the viral envelope $(28,39)$. The S RNA encodes a nonstructural (NSs) protein, which forms filamentous inclusion bodies (20), in the $\mathrm{v}$ sense, and a nucleocapsid $(\mathrm{N})$ protein, which encapsidates viral RNAs $(7,24)$, in the vc sense.

Watermelon silver mottle virus (WSMV), causing severe damage on watermelon in Taiwan, was previously identified as a distinct tospovirus based upon particle morphology, host reactions, thrips transmission, and serological relationships (43). The N protein of WSMV is serologically unrelated to tomato spotted wilt virus (TSWV) and impatiens necrotic spot virus (INSV) and has a

Corresponding author: S.-D. Yeh; E-mail address: sdyeh@nchu.edu.tw

Publication no. P-1998-0216-01R

(C) 1998 The American Phytopathological Society larger molecular mass of $30.7 \mathrm{kDa}(1,42,43)$. The strong serological cross-reactions (1) observed among a watermelon isolate (Tospo$\mathrm{W}$ from Taiwan [43]), a tomato isolate (Tospo-To from Taiwan [1]), an isolate causing silver mottle disease on watermelon in Japan (17), and a groundnut isolate from India designated peanut bud necrosis virus (PBNV) $(11,31)$ placed these viruses together as a distinct serogroup IV of tospoviruses (1). Recently, the complete nucleotide sequences of the S RNA of the watermelon isolate Tospo-W $(42,44)$ and the tomato isolate Tospo-To (14) have been determined. The amino acid sequences of their $\mathrm{N}$ proteins share 99\% identity, indicating that they are actually two closely related strains of WSMV. Comparison of the N protein of WSMV with those of TSWV (serogroup I), groundnut ringspot virus (GRSV) (serogroup II), tomato chlorotic spot virus (TCSV) (serogroup II), and INSV (serogroup III) revealed fairly low amino acid identity (26.4 to $33.3 \%$ ), indicating that WSMV is phylogenetically distinct from tospoviruses of serogroups I, II, and III (42). The S RNA of the India PBNV is 3,057 nucleotides in length with an intergenic region 488 nucleotides shorter than WSMV, and its $\mathrm{N}$ protein shares $86 \%$ amino acid identity with that of WSMV (35, 44). Moreover, WSMV induces severe systemic symptoms in most cucurbit species and does not infect peanut, while PBNV causes systemic infection in peanut but local infection in cucurbits $(31$, 42). Although the close serological relationship between the two $\mathrm{N}$ proteins suggests that WSMV and PBNV are two strains of the same species in serogroup IV $(1,31)$, the differences in the S RNA and the distinct host reactions imply that they are two related and yet distinct tospovirus species (35).

In this investigation, the dsRNAs of WSMV, INSV, and TSWV extracted from virus-infected hosts were compared, and the nucleotide sequence of the genomic M RNA of WSMV was determined from the cDNA clones derived from the separated M dsRNA. 
The complete nucleotide sequence of the M RNA was compared with those of INSV (25), TSWV (18), and PBNV (36) to further clarify the taxonomic position of WSMV. The existence of the ambisense expression strategy is supported by the presence of specific RNA messages in WSMV-infected tissue detected by strandspecific probes.

\section{MATERIALS AND METHODS}

Virus sources and propagation. An isolate of WSMV collected from watermelon (Citrullus lanatus (Thunb.) Matsum. \& Nakai) in Taiwan, previously designated as TSWV-W or Tospo-W (42-44), was used for the study. A typical isolate of TSWV that originated from tomato in New York State (denoted TSWV-NY, provided by R. Provvidenti, New York State Experiment Station, Geneva) and a typical isolate of INSV collected from impatiens in the United States (denoted INSV-M, given by J. Moyer, North Carolina University, Raleigh) were also used for comparison.

The three viruses were maintained in the systemic host Nicotiana benthamiana Domin. and the local lesion host Chenopodium quinoa Willd. by mechanical transfers. The inoculum was prepared by grinding infected leaf tissue in $0.01 \mathrm{M}$ potassium phosphate buffer ( $\mathrm{pH} 7.0$ ) containing $0.01 \mathrm{M}$ sodium sulfite.

Isolation of total RNA and dsRNA. WSMV was propagated in the systemic host $N$. benthamiana, and leaves were harvested 4 to 6 days after mechanical inoculation. Total RNA was extracted from healthy and WSMV-infected leaves according to the method of Verwoerd et al. (41). For isolation of viral dsRNAs, WSMV, INSV, TSWV, and tobacco mosaic virus (TMV) were propagated in the local lesion host $C$. quinoa, zucchini yellow mosaic virus (ZYMV) in the systemic host zucchini squash (Cucurbita pepo L.), and cucumber mosaic virus (CMV) in tobacco ( $N$. tabacum L.). The procedure for isolation of viral dsRNAs was similar to that described by Yeh and Chang (42). The dsRNAs were eluted from a CF-11 cellulose column and precipitated by ethanol. The $\mathrm{M}$
dsRNA was separated by electrophoresis (Fig. 1), eluted from agarose gel, and used for the construction of a cDNA library.

Construction of the cDNA library from $M$ dsRNA and hybridization selection. The $M$ dsRNA was denatured by methylmercuric hydroxide ( $\mathrm{MeHg}$ ) (2). The cDNA library was constructed according to the protocols for ZAP-cDNA synthesis and cloning (Stratagene Inc., La Jolla, CA). The methylated firststrand cDNA was synthesized by Moloney murine leukemia virus reverse transcriptase after the addition of random primers. The second-strand cDNA was generated according to Gubler and Hoffman (13) by RNase $\mathrm{H}$ digestion and DNA polymerase I extension. The ds cDNA was filled in with $P f u$ DNA polymerase (Stratagene Inc.) and ligated with EcoRI adaptors at both ends. The dsDNA was inserted into the lambda ZAP-II EcoRI/calf intestinal alkaline phosphatase (CIAP) vector (Stratagene Inc.) and packaged with capsid proteins (Gold Packaging Extract; Stratagene Inc.).

The cDNA clones were screened by hybridization according to the method of Maniatis et al. (26). The cDNA probe used for plaque screening was synthesized according to Maniatis et al. (26), using ${ }^{32} \mathrm{P}$-labeled cDNA synthesized from MeHg-denatured M dsRNA by random priming.

Cloning the $5^{\prime}$ - and $3^{\prime}$-terminal regions of $v$ M RNA. cDNA clones corresponding to the $5^{\prime}$ and $3^{\prime}$ ends of the $\mathrm{v}$ strand of the $\mathrm{M}$ RNA were obtained by reverse transcription-polymerase chain reaction (RT-PCR) with specific primers. The $M$ dsRNA of WSMV was denatured by boiling for $5 \mathrm{~min}$ with a first downstream primer (nsm3 of 5'-CGTCCCACTATTCCATGTAGGC-3', nucleotide positions 446 to 427; g1 of $5^{\prime}$-GGACTAGTTGTTGCTCTGGACATGGG-3', positions 3,361 to 3,378; or g3 of 5'-GAGAAGCATCCACAGATCCC-3', positions 3,770 to 3,789 ). The first-strand cDNA was synthesized by SuperScript II reverse transcriptase (Gibco BRL, Gaithersburg, MD). After removal of template RNA by RNase $\mathrm{H}$ digestion, the first-strand cDNA was tailed with dCTP and terminal deoxynucleotidyl transferase (Gibco BRL). PCR amplification of the deoxycytidine-tailed cDNA was per-

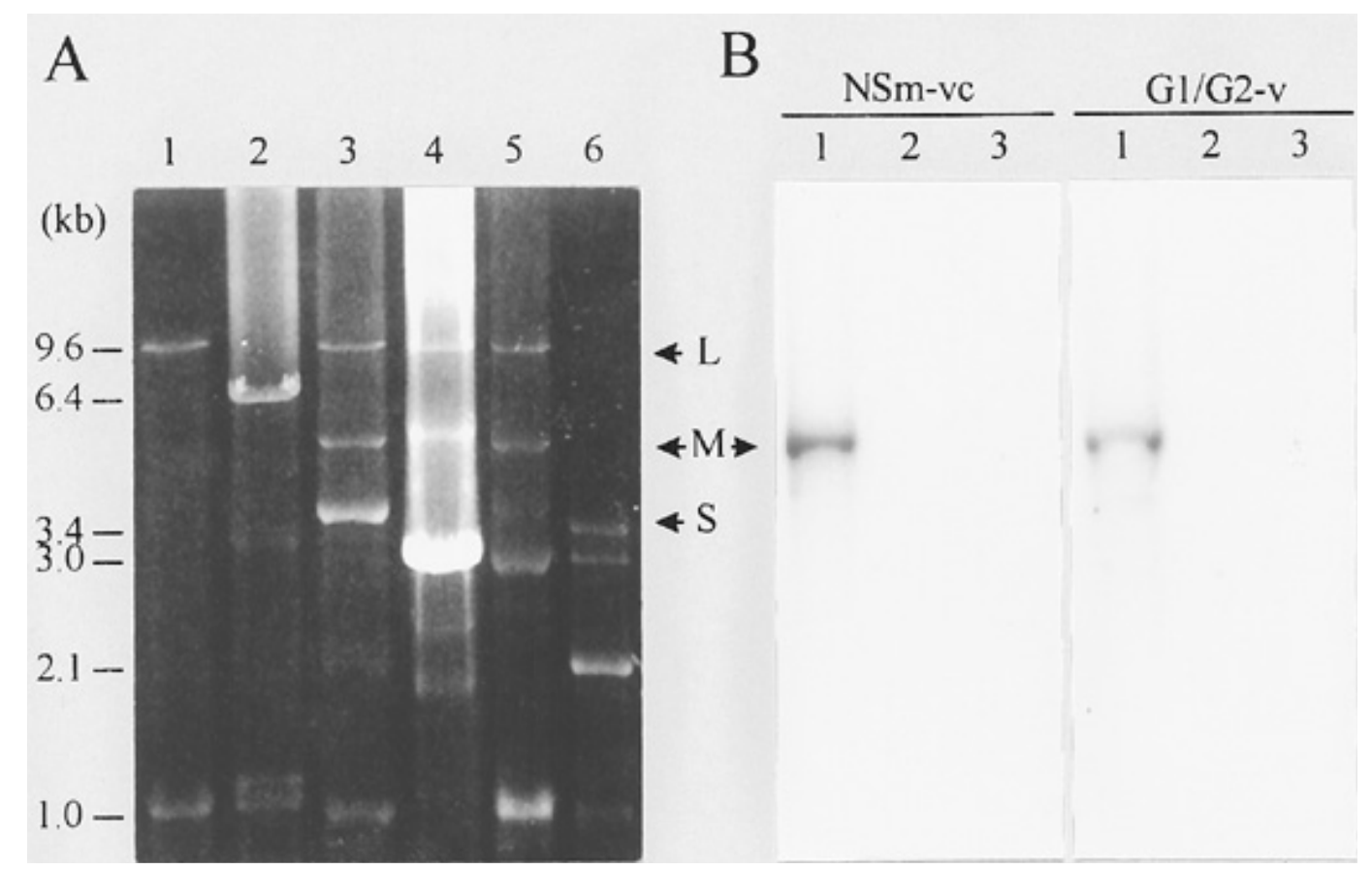

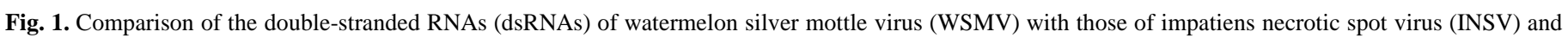

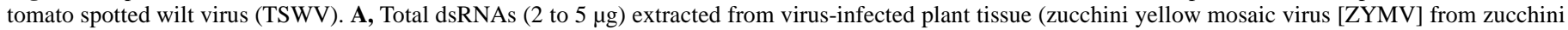

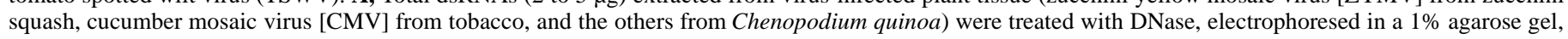

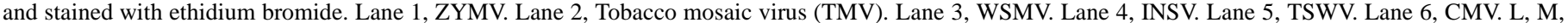

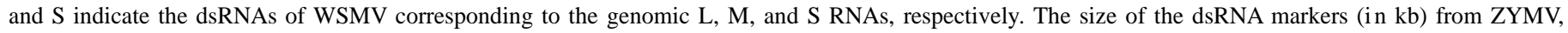

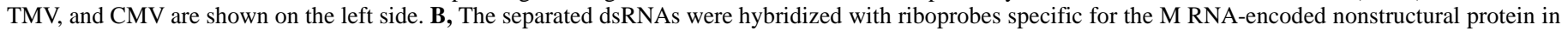
the viral complementary sense (NSm-vc) and G1/G2 (G1/G2-v) open reading frames of WSMV. Lane 1, WSMV. Lane 2, INSV. Lane 3, TSWV. 
formed with Taq DNA polymerase (TaKaRa, Shiga, Japan), using an upstream primer (G12) of 5'-CGACTAGTGGGGGGGGGGGG-3' containing a SpeI site and a second downstream primer (nsm1 of 5'-ATTCACTGGAGTAGGTCCC-3', positions 279 to 259; g2 of 5'-CGCACTCGCATGTTTCATACCC-3', positions 3,425 to 3,444 ; or $\mathrm{g} 4$ of 5'-TGCCCATTACATCGTACC-3', positions 3,852 to 3,869 ). The corresponding positions of the primers on the $\mathrm{v}$ strand of the $\mathrm{M}$ RNA are indicated in Figure 2. The amplified cDNAs were directly inserted into the pT7 Blue T-vector (Novagen, Inc., Madison, WI) to generate recombinants that contained the amplified cDNA fragments from the $5^{\prime}$ - and $3^{\prime}$-terminal regions of the v strand of the M RNA.

DNA sequencing and data analysis. DNA sequencing was performed by the dideoxynucleotide chain-termination method (34), using ssDNA templates generated by VCSM13 (Stratagene Inc.) or dsDNA templates prepared from selected clones (16). The sequencing method using T7 DNA polymerase followed the instructions for the Sequenase version 2.0 DNA sequencing kit (United States Biochemical Corp., Cleveland). Sequence data were assembled and analyzed using PC/GENE software (version 6.85; IntelliGenetics, Inc., Mountain View, CA). The aligned sequence reflecting the complete $\mathrm{v}$ strand of the M RNA of WSMV was compared with those of INSV (25), TSWV (18), and PBNV (35).

Purification of nucleocapsids and extraction of viral RNAs. Leaves of WSMV-infected C. quinoa were harvested 4 to 6 days after inoculation and homogenized with the extraction TB buffer (0.01 M Tris- $\mathrm{HCl}, \mathrm{pH} 8.0 ; 0.01 \mathrm{M}$ sodium sulfite; and $0.1 \%$ cysteine) in a Waring blender (Waring, New Hartford, CT) for $1 \mathrm{~min}$ ( 3 to $4 \mathrm{ml} / \mathrm{g}$ of tissue). After filtering through four layers of cheesecloth, the extract was centrifuged at $15,300 \times g$ for $10 \mathrm{~min}$. The supernatant was stirred with $1 \%$ Triton X-100 (Sigma Chemical Co., St. Louis) (final concentration) at $4^{\circ} \mathrm{C}$ for $5 \mathrm{~min}$, and then centrifuged at $79,700 \times g$ for $120 \mathrm{~min}$ through a $20 \%$ sucrose cushion. The pellets were then resuspended in TBG buffer (TB buffer containing $0.01 \mathrm{M}$ glycine) and further purified by $35 \%$ cesium sulfate isopycnic centrifugation at $83,500 \times g$ for $16 \mathrm{~h}$. The opalescent zones containing virus nucleocapsids were collected, further concentrated by centrifugation at $163,600 \times g$ for $75 \mathrm{~min}$, and resuspended in TBG buffer as purified nucleocapsids. Viral RNAs were extracted from nucleocapsids by the sodium dodecyl sulfate-phenol method as described by Schwinghamer and Symons (37).

Northern blot analysis. The dsRNAs extracted from $15 \mathrm{~g}$ of tissue were treated with DNase (10 U; Stratagene Inc.) at $37^{\circ} \mathrm{C}$ for $10 \mathrm{~min}$ and analyzed by electrophoresis on nondenaturing TBE (0.089 M Tris-HCl, 0.089 M sodium borate, and 0.002 M EDTA) $1 \%$ agarose gels. For hybridization analysis, the dsRNAs were denatured in the gel with $100 \mathrm{mM} \mathrm{NaOH}$ for $5 \mathrm{~min}$ and neutralized in $0.5 \mathrm{TBE}$ for $60 \mathrm{~min}$ prior to transferring (25) to nylon membranes. The viral RNAs extracted from nucleocapsids and total RNA extracted from WSMV-infected $N$. benthamiana were separated by electrophoresis in a $1 \%$ formaldehyde denaturing agarose gel and then transferred to nylon membranes (33). The membranes were hybridized with strand-specific RNA probes prepared from clones containing parts of the NSm (clone MP, nucleotide positions 21 to 911, Fig. 2) and G1/G2 ORFs (clone M13, nucleotide positions 2,311 to 3,500, Fig. 2). The ${ }^{32} \mathrm{P}$-labeled transcripts with polarity of the vc sense of the NSm gene or the $\mathrm{v}$ sense of the G1/G2 gene (probes NSm-vc and G1/G2-v, respectively) were synthesized using $\mathrm{T} 3$ polymerase, and those with polarity of the $\mathrm{V}$ sense of the NSm gene or the vc sense of the G1/G2 gene (probes NSm-v and G1/G2-vc, respectively) were synthesized using T7 RNA polymerase, as described by the manufacturer (Epicentre Technologies, Madison, WI). Hybridization conditions followed the protocols of Amersham Corp. (Arlington Heights, IL).

\section{RESULTS}

Comparison of dsRNA patterns and hybridization analysis. The yields of the total dsRNA segments of WSMV extracted from virus-infected leaf tissue of $C$. quinoa ranged from 50 to $120 \mu \mathrm{g}$ per $15 \mathrm{~g}$ of tissue. After DNase treatment, a typical pattern with three classes of dsRNA segments of WSMV was obtained (Fig. 1A, lane 3). dsRNAs extracted from ZYMV-infected zucchini squash (9.6 kbp), TMV-infected C. quinoa (6.4 kbp), and CMV-infected tobacco $(3.4,3.0,2.1$, and $1.0 \mathrm{kbp})$ were used as molecular markers (Fig. 1A, lanes 1, 2, and 6, respectively). dsRNAs extracted from leaves of $C$. quinoa infected with INSV-M or TSWV-NY (Fig. 1A, lanes 4 and 5, respectively) were compared with those of WSMV. The ds forms of the genomic L, M, and S RNAs of WSMV were estimated as 9.0, 4.9, and $3.5 \mathrm{kbp}$, respectively. The M dsRNA of WSMV was slightly shorter than that of INSV-M (Fig. 1A, lane 4) and slightly longer than that of TSWV-NY (Fig. 1A, lane 5). The length of the L RNA of WSMV was similar to those of INSV and TSWV. However, the S dsRNA of WSMV was much longer than those of INSV and TSWV, in agreement with the previous reports that the S RNA of WSMV is $0.6 \mathrm{~kb}$ longer than that of INSV and $0.75 \mathrm{~kb}$ longer than that of TSWV $(14,44)$.

When dsRNAs of WSMV, INSV-M, and TSWV-NY were hybridized with strand-specific RNA probes prepared from clones corresponding to the NSm and G1/G2 ORFs (NSm-vc and G1/G2$\mathrm{v}$, respectively), each of the probes reacted strongly with the $\mathrm{M}$ dsRNA of WSMV, but failed to hybridize with the M dsRNAs of INSV-M and TSWV-NY (Fig. 1B). This indicated that the M RNA of WSMV is phylogenetically distinct from those of INSV$\mathrm{M}$ and TSWV-NY, with homology insufficient for hybridization under the conditions of Figure 1B.

Cloning and sequence determination of WSMV M RNA. Three clones (M3, M6, and M13) were selected by hybridization with cDNA probes derived from the M dsRNA of WSMV. These clones contained most of the M RNA segment, but with the $5^{\prime}$ and $3^{\prime}$ ends missing (Fig. 2). The largest cDNA clone, M6, was digested with EcoRI and subcloned into pBluescript II SK(-) vector (Stratagene Inc.) to generate two clones, ML and MS. The sequences of the M6 and ML clones were determined using a nested-deletion strategy (15). The sequence of the subclone MS was verified by sequencing in both directions. Three subclones, MP (PstI-digested), MX (XbaI-digested), and MX-ve (PstI- and nest-digested), were derived from clone M3 (Fig. 2).

The 0.28 -kbp cDNA fragment, amplified by the G12 and nsm1 primers and corresponding to the 5'-terminal region of $\mathrm{v} M$ RNA, was directly inserted into the pT7 Blue T-vector and the clone NSM1 obtained was sequenced in both directions. The cDNA clone G2 amplified with G12 and g2 primers and corresponding to the 3 '-terminal region of v M RNA was obtained and sequenced. As the $\mathrm{G} 2$ clone did not extend to the $3^{\prime}$ terminus, the designed primers may have bound within the $3^{\prime}$ part of the $\mathrm{v}$ M RNA and blocked extension by reverse transcriptase. Thus, two primers, g3 and $\mathrm{g} 4$, designed from the extended sequence of the $\mathrm{G} 2$ clone and

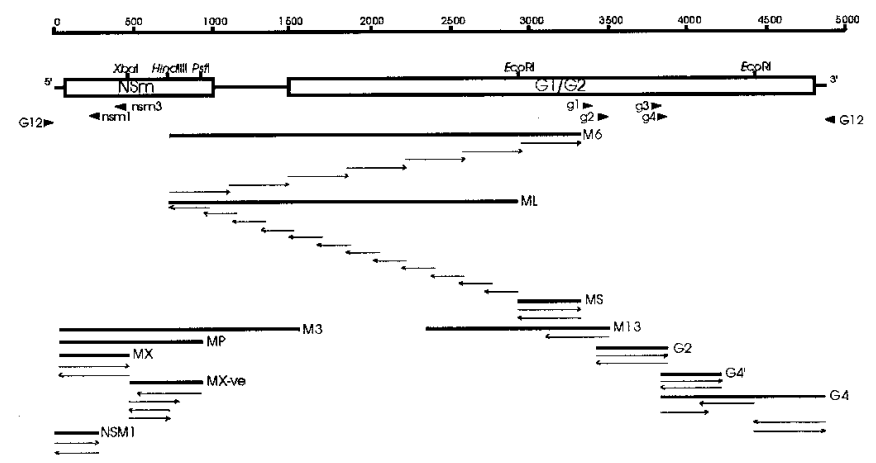

Fig. 2. Strategy for cDNA cloning and sequence determination of watermelon silver mottle virus M RNA. The clones are denoted by thick lines. Arrows indicate the direction of sequencing. Arrow heads indicate the primers used for reverse transcriptase-polymerase chain reaction amplification of the $5^{\prime}$ and $3^{\prime}$ ends of the M RNA. 
AGAGCAATCGGTGCGCCAATTACTAGTTAAATCATCAAATAATTAACAAGAAATAATGTATCGCT TGTCTAATGTCTTAAAACCTTTCCGTCTT TCGAATAACACAAATAAAGAGATGGT

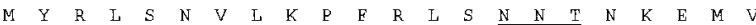
CCCTGCTGTGAAAACGGAAAACAACAAGAGCATTATAGTTAGAAATGTCTCTAAAAAGGACATAGATGAGGCTATAATGA.ACAAAGTTAAGACAGCAAATGGGAAACAATATGTTTCTGG

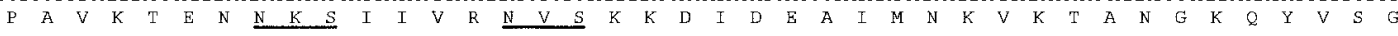
CATAGATTCTAGTATCTIGGGACCTACTCCAGTGAATTAGATACAGGTGAGTCAGATGTAGGAGACAGTICAGATGATATTTTATCCAGACT TGTAGTTGAACAAAGCACCCACTTGAG

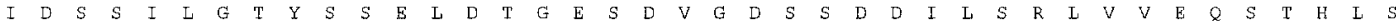
CAATTGGAAAAA.TGACTCTCTTGTTGGTAATGGAAATGACAAAGTCAGTTTCACTATAAGCATAATGCCTACATGGAATAGTGGGA.GGAGATTCATGCATATATCTAGACTCATATTGTG

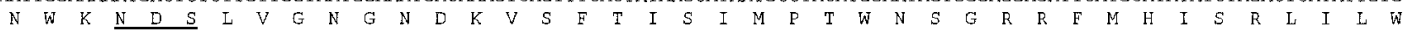
GGTTGTCCCAACTATTCCCGATTCAAAGAACAGCATCAAGGTCACTCTACTCGATCAAAACAAAATGACTCGTGAAGAGAAGATAATTTTGAGCAGGCAAACATCTTTAAAAGATCCTTT

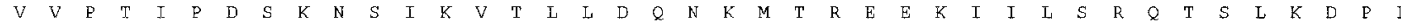
GTGCTTCATTTTCACTTGAATTGGTCTTCCCTAAAGAAAGAATACACCTAAGCAGTGCATGCAACTTAACCTGACAAGTGATGAAAAGTATGCTAAAGGGGTAGCT TIGCATCAGT

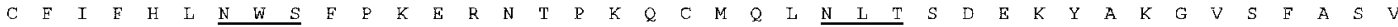
TATGTATTCCTGGGTAAAAAACTTCTGTGATACTCCTATTGCATCAGAAAGTAATACTTGTGATGTGATACCAATAAATAGAGCTAAGGTAATTAAATCTGCTGCTTTGATTGAAGCATG

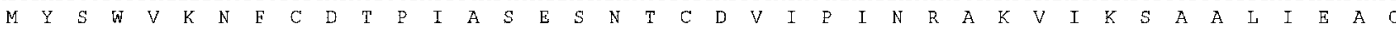
TAAGCTAATGATACCAAAGGGAAT TGGAGGGAAGCAACTATCGAACCAAATTAGAGCTCTACAAAAGGCTGCAGAAAAATATGCAATGGAAGCAGAGAATGATGCAGATGTCATTGATGT

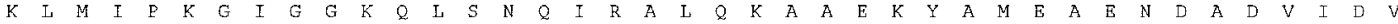
GGATATAGAAATGGATAACCTTCTTGAAATTTAAATCGTAAGCAAATGATTTAGGCTAT TTAAATATTAATTTGTGTTTGAATTTAGATTTAAACATAATTAAATCTATTTTCTTGTGTG D I E M D N L L E I *

CTTATGAGTCAGTATTTTATATTTTGPATTTGTCTCTATGTATGTCTAGAATATGTATAGTTGTATGCAATTTTAATAATAATAAATAAATACATAAAAACAACAAAAATATGCAAAA

ATCAAAAACAAAAAATATATATATATAT AAAATAAAAATAAAATAATAACTAACTATTATATAAT TAAATAAGGCTACGGCCARGTGT TGGCTTTTCAGCCT TTTTTTGCTATTTTTTA TTTTCTAGTTTTTTTGTTTATT TGTTTT TATCATAGTTTTATTTGTTTCATTTATTATTTTACTCATCTACTTACTTATCTGACTTTTTCATGCTGTGTAGCTGAGCGCAATTRATTTTC

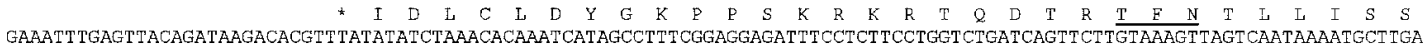

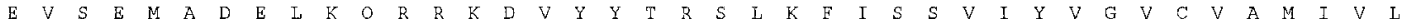
TTCAACAGACTCCATAGCAT CTTCTAAT TTCTGCCT TCTTTTATCTACGTAGTATGTTCTAGATAATTTGAATATGCTGCTCACAATATATACACCCACACACACAGCCATGATCACTAA

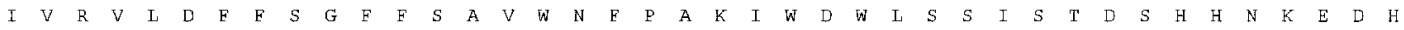
AATTACTCTAACTAAATCAAAGAAACTACCGAAGAAAGAAGCTACCCAAT TAAAAGGTGCTTTGATCCAGTCCCACAAGCTAGAAATGGAAGTGTCAGAATGGTGCTTGTTT TCATCAT

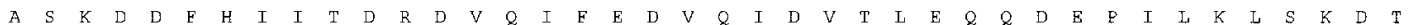
TGCACTTTTGTCATCAAAGTGAATTATTGTGTCTCGATCAACCTGAATGAACTCATCTACT TGTA.TGTCTACTGTAAGTTCTTGTTGATCTTCAGGGATGAGTTTAAGAGATTIGTCTG

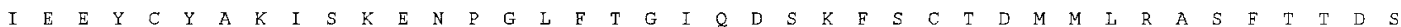

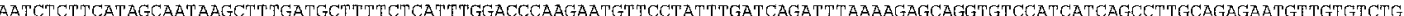

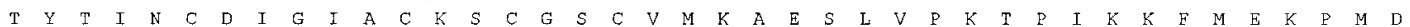
CGTATAAGTTATAT TGCAATCAATCCCTATAGCACATTTTGAGCATCCAGAGCACACCATTTTAGCCTCTGAAAGGACAGGTTTGGTTGGTATT TTTTTGAACATTTCTTTGGGCATATC

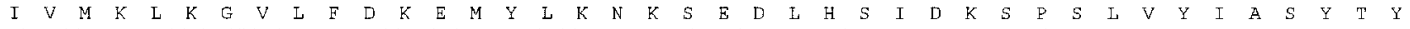
TATAACCATT TTAAGCTTACCGACTAAAAAATCCTTCTCCATATACAGCTTATTTTTGGATTCATCTAGATGTGATATGTCTTTTGATGGAGATAAAACATAAATAGCACTGTATGTGTA

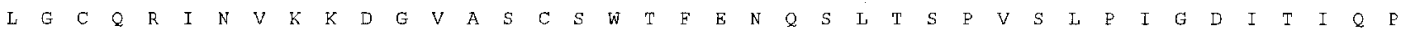
CAGTCCGCATTGCCTTATGTTAACTTTTTTGTCTCCTACAGCACTGCAACTCCATGTAAACTCAT TTTGAGATAAAGTGGATGGAACTGATAACGGGATGCCATCTATAGTTATCTGCGO

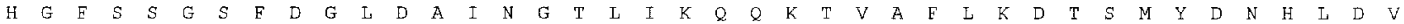
ATGACCAAATGATGATCCTGAAAAATCACCCAAATCAGCAATATTCCCTGTGAGAATTTCTGCTGT TTCGTGACAGCAAATAACT TATCAGTACT CATATAATCATTGTGTAGATCCAC

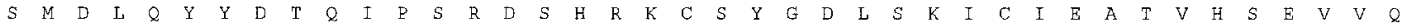
TGACATGTCAAGTTGATAATAATCTGTT TGAATAGG TGACCTATCAGAATGTCTCTTGCAAGAATAACCATCTAATGAITTTATACAAATTTCCGCAGTAACATGACT TTCCACAACC TG

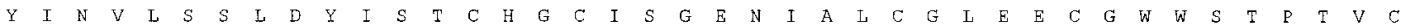
GTAAATGTTGACTAGACTTGAT AAATCATAAATGCT TGTGCAATGGCCACAGATGGATCCTTCATTGATTGCTAAACAGCCAAGCTCT TCACATCCCCACCAAGAAGT TGGGGTAACGCA

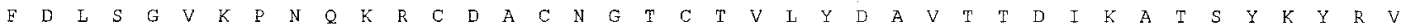
AAAATCTAACGATCCTACTTTAGGGTTTTGCTTTCTACAGTCTGCACAATTGCCTGTGCATGTGACCAAATAATCTGCTACTGTTGTATCAATTTT TGCCGT TGAGTACT TGTATCTTAC

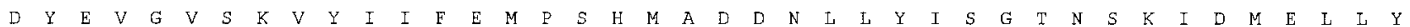
ATCATAITCAACACCAACACTT TTTACATAAATTATGATTCCATTGGGGAGTGCATAGCATCATCGTTAAGCAAATATATCGATCCTGTGTTAGATTTAATGTCCATTTCTAAAAGATA

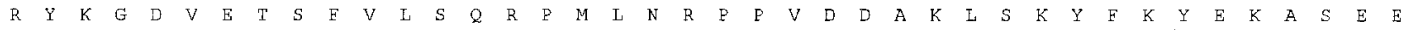
TCTATATTTCCCATCAACCTCTGTTGAAAATACTAAAGATTGCCTAGGCATCAAATTTCTAGGTGGAACATCATCTGCTTTTAAACTCTTATAAAATTTGTATTCCTIGGCAGATTCT TC

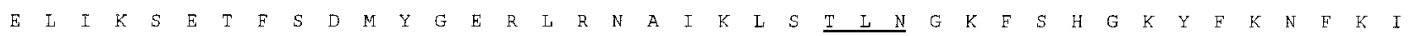

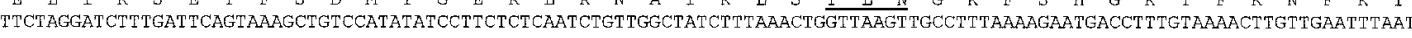

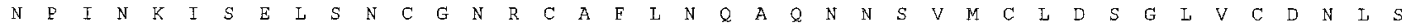
ATITGGTATGITTT TTAT GGACTCAAGGGAGTTACAGCCATT TCTGCATGCAAACAAGTTITGAGCT TGATTGTTGCTCACCATACACAGATCTGAGCCTIAAAACACAATCGTPTAGCGA

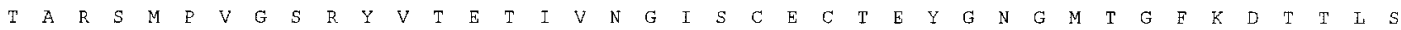
TGTTGCTCTGGACATGGGTACACCAGATCTGTACACCGTTTCAGTAATAACATTCCCTATTGAACACTCGCATGTTTCATACCCATTACCCATTGTTCCAAACTTGTCTGTTGTCAAAGA

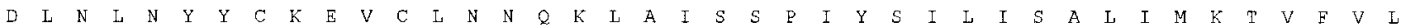
ATCTAAATTCAAATTGTAATAACATTTTTCCACGCACAAGITGTTCTGTTTGAGTGCAATACTTGACGGTATGTAAGAGATAAGAATTGATGCCAGATCATTTTTGT TACAAAAACCAG

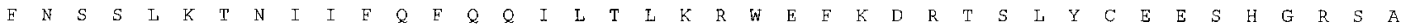
AAAATTGCTACTTAACTTGGTGTTTATTATAAACTGAAATTGCTGGATCAAAGTCAATTTGCGCCATTCAAATTTATCTCTGGTGGATAGATAGCATTCT TCAGAATGACCTCTAGATGC

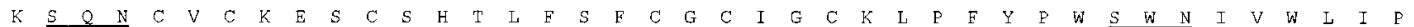
TTIGCTTGATPACAAACACACTrTTCAGAGCAAGAATGAGT TAAGAAGGAGAAGCATCCACAGATCCCACAT TTTAGAGGGAAATATGGCCAT GACCAAT TAATGACCCAAAGGATAGS

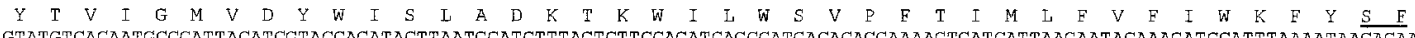
GTATGTCACAATGCCCATTACATCGTACCAGATACT TAATGCATCTTTAGTCTTCCAGATCAGCCATGACACAGGAAAAGTGATCATTAAGAATACAAAGATCCATTT AAAATAAGAGA

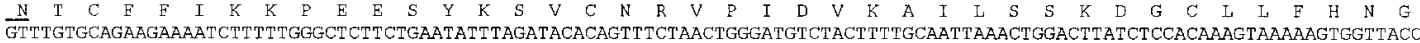

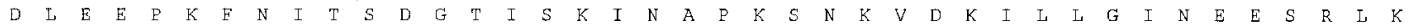
ATCTAGTTCTTCAGGTTTAAAGTTTATTGTIGAGTCACCAGTAATTGATTTTATGTTTGCAGGTTTAGAGTTCTTTACATCCTTTATCAGCAACCCTATGTTCTCTTCTGATCT CAATTT

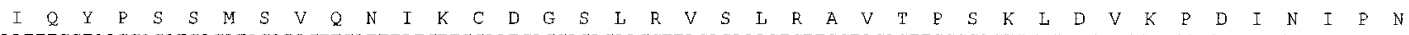
AATTIGGTAAGGAGATGACATAGATACT TGATTTATCT TGCAATCACCAGACAACCTTACAGAAAGTCTTGCTACAGTTGGGGACTTCAGATCCACCTTCGGGT CAATGT TGATCGGGTT

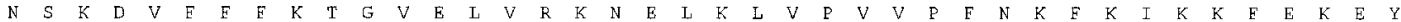
GTT TGATTTATCAACGAAGAAAAATTITGTTCCCACTTCAAGACTCTTTTGTTTTCCAGTTTCAGAACAGGGACCACTGGGAAATITITGAACTTGATTIT CT TGAATTCTTTTTCATA

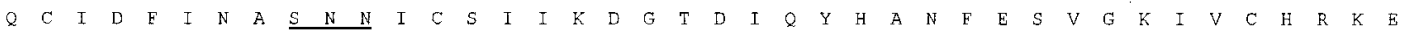
CTGGCAAATATCAAATATAPTAGCAGARTTGTTAATGCATGAGATAATTTTATCACCGGTATCTATT TGGTAATGGGCATTGAACTCGCTCACACCTTTGATGACACAGTGTCTTITTTC

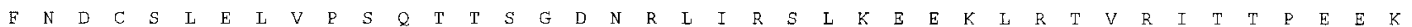
AAAATTGTCACAACTTAATTCTAGGACAGGAGACTGTGTTGTGCTCCCATCGTTCT TAGATCCGTGACAATTCCTCTTCCTGAGTCTAGTTACTCTGAT TGTTGTGGGTTCCTCTTT

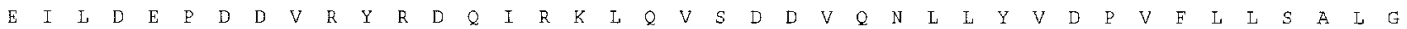
CTCAATGAGGTCTTCAGGGTCGTCTACTCTGTATCTATCT TGGATTCT TT TTAACTGGACACTGTCATCCACCTGGTTCAAAAGGTAGACATCAGGGACGAACAAAAGCGAAGCCAGACC $\begin{array}{llllllllllllllllll}L & C & X & V & L & L & Y & Y & K & K & M\end{array}$ TAGACAGTAGACAAGTAGATAGTATTTCTTCATTCTTAAAAGGT TCTTTGAGAGTATTTAATTGTTGCACTGATTGCTCT 4880

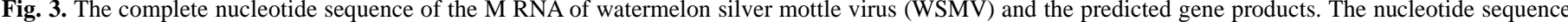

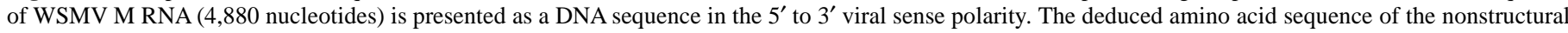

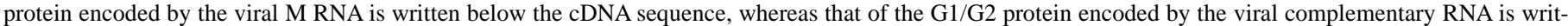
ten above the cDNA sequence. The asterisks indicate termination codons. Potential $N$-glycosylation sites are underlined. 
paired with the primer G12 were used for RT-PCR to obtain an amplified cDNA fragment containing the 3'-terminal region of the $\mathrm{v}$ M RNA. Two cDNA fragments of 0.4 and $1 \mathrm{kbp}$ were obtained using the $\mathrm{G} 12$ and $\mathrm{g} 4$ primers and were used to generate the $\mathrm{G} 4{ }^{\prime}$ and $\mathrm{G} 4$ clones, respectively.

The sequence assembled with the above clones reflected the complete genomic M RNA of WSMV. The complete nucleotide sequence of the v M RNA of WSMV contained 4,880 residues as shown in Figure 3. It had a base composition of $31.6 \%$ A, 33.5\% $\mathrm{U}, 17.7 \% \mathrm{C}$, and $17.2 \% \mathrm{G}$. Computer prediction for the possible reading frames of the $\mathrm{v}$ and $\mathrm{vc}$ strands of the M RNA revealed an ambisense coding strategy similar to that of the reported M RNAs of other tospoviruses $(18,25,36)$, having a large ORF in the vc strand coding for a G1/G2 precursor protein of 1,121 amino acids with a calculated molecular mass of $127.6 \mathrm{kDa}$ and a small ORF in the $\mathrm{v}$ strand coding for a NSm protein of 312 amino acids (35.0 $\mathrm{kDa}$ ). The ORFs were separated by a 473-nucleotide A-U-rich intergenic region. The $5^{\prime}$ and $3^{\prime}$ noncoding sequences of WSMV M RNA were 55 and 47 nucleotides in length, respectively.

Comparison of the M RNA of WSMV with other tospoviruses. Comparison of the complete nucleotide sequence of the genomic M RNA of WSMV with those of TSWV (18), INSV (25), and PBNV (36) is summarized in Figure 4. The sequence data were in agreement with the dsRNA pattern in that the M dsRNA of WSMV was slightly longer than that of TSWV and slightly shorter than that of INSV (Fig. 1A). The M RNA of WSMV had an overall nucleotide identity of 56.4, 56.9, and 79.6\% with those of TSWV, INSV, and PBNV, respectively.

The $5^{\prime}$ and $3^{\prime}$ noncoding sequences of WSMV M RNA (55 and 47 nucleotides in length, respectively) were similar to those of PBNV (56 and 47 nucleotides, respectively), but shorter than those of TSWV (100 and 84 nucleotides, respectively) and INSV (85 and 169 nucleotides, respectively). The $5^{\prime}$ noncoding region of WSMV M RNA shared a nucleotide identity of $85.5,78.2$, and 94.6\% with those of TSWV, INSV, and PBNV, respectively. The 3' noncoding region of WSMV M RNA shared 76.6, 78.7, and $85.1 \%$ identity with those of TSWV, INSV, and PBNV, respectively. The 5'- and 3'-terminal regions of the M RNA of WSMV contained inverted complementary sequences, similar to terminal sequences found in other tospoviruses $(18,25,36)$. There were $74.5 \%$ complementary residues between the $5^{\prime}$ and $3^{\prime}$ noncoding sequences of WSMV M RNA, and they could form a stable panhandle structure. The terminal 26 nucleotides at the $5^{\prime}$ and $3^{\prime}$ ends of the $M$ RNA of WSMV, respectively, were identical to those of PBNV M RNA. Thirteen of the first terminal 14 nucleotides (except the tenth position) were completely complementary. The $5^{\prime}$ termini of the S and M RNAs of WSMV had an identical sequence of 10 nucleotides, and their $3^{\prime}$ termini had an identical sequence of nine nucleotides. The $5^{\prime}$ terminus of the M RNA of WSMV shared a consensus sequence of nine nucleotides in common with those of TSWV and INSV, and the $3^{\prime}$ terminus a sequence of 16 nucleotides. Both termini of WSMV M RNA shared 26 nucleotides in common with those of PBNV. Comparison of the $5^{\prime}$ and $3^{\prime}$ ends of the $\mathrm{S}$ and $\mathrm{M}$ RNAs of all four tospoviruses revealed a consensus sequence of eight nucleotides (5'-AGAGCAAU...-3') at the $5^{\prime}$ ends and a consensus sequence of eight nucleotides $\left(5^{\prime}\right.$-...AUUGCUCU-3') at the $3^{\prime}$ ends.

The NSm ORF started at nucleotide position 56 and terminated at nucleotide position 994. Analysis of the amino acid sequence did not reveal any hydrophobic regions that might function as signals or transmembrane spanning segments. There were six $N$-glycosylation sites predicted (Fig. 3) that were identical to those of PBNV, but it is not known whether they are used in vivo. There was only one glycosylation site at amino acid positions 189 to 191 (NWS) identical to those of TSWV and INSV. The NSm ORF was 30,27 , and 15 nucleotides (10, 9, and 5 amino acids, respectively) longer than those of TSWV, INSV, and PBNV, respectively. Comparison of the nucleotide sequence of the WSMV NSm ORF with those of TSWV, INSV, and PBNV revealed that they shared an identity of $55.4,55.5$, and $77.4 \%$, respectively. The amino acid sequence of the WSMV NSm protein shared identity of 34.4, 40.9 , and $83.4 \%$ with those of TSWV, INSV, and PBNV, respectively. The N-terminal regions of the NSm proteins (first 65 amino acids) of the four tospoviruses compared were found more variable than the central and $\mathrm{C}$-terminal regions.

The RNAFOLD program (PC/GENE) (45) predicted two major stem-loop structures in the A-U-rich intergenic region (data not shown) that may be signals for transcription termination of the NSm and G1/G2 ORFs within the ambisense M RNA segment of WSMV, as speculated for INSV (25) and TSWV (18). The intergenic region of WSMV M RNA was the same length as that of INSV (473 nucleotides), but 153 and 64 nucleotides longer than those of TSWV and PBNV, respectively. The nucleotide sequence of the intergenic region shared 50.3, 59.0, and 62.5\% identity with that of TSWV, INSV, and PBNV, respectively.

The G1/G2 ORF in the vc strand of WSMV M RNA, ranging from positions 1,468 to 4,833 (numbered from the $5^{\prime}$ end of the viral RNA), was 3,366 nucleotides in length. There were seven putative $\mathrm{N}$-glycosylation sites predicted by the PROSITE program (3) located in this protein (Fig. 3), four of which were identical to PBNV and only one (NFS, amino acid positions 291 to 293) was found conserved among the four tospoviruses compared. Three membrane-spanning segments were predicted by the program RAOARGOS (30) to occur at amino acid positions 286 to 313, 410 to 429 , and 1,036 to 1,068 and were found in all four tospoviruses compared. Four transmembrane segments were predicted by the program SOAP (22). The cell attachment site RGD has been predicted to occur on other animal virus surface glycoproteins and may be involved in cell adhesion (32). The RGD motif, which is present in the INSV and TSWV glycoprotein precursors $(18,25)$ but absent in PBNV (36), was not found in WSMV. The G1/G2 ORF was the same length as in PBNV, but 33 nucleotides longer than that of INSV and 42 nucleotides shorter than TSWV. Comparison of the nucleotide sequence of the WSMV G1/G2 ORF with that of TSWV, INSV, and PBNV revealed that they shared an identity of $56.3,55.6$, and $81.4 \%$, respectively. The amino acid sequence of the WSMV G1/G2 protein shared an identity of 32.2,
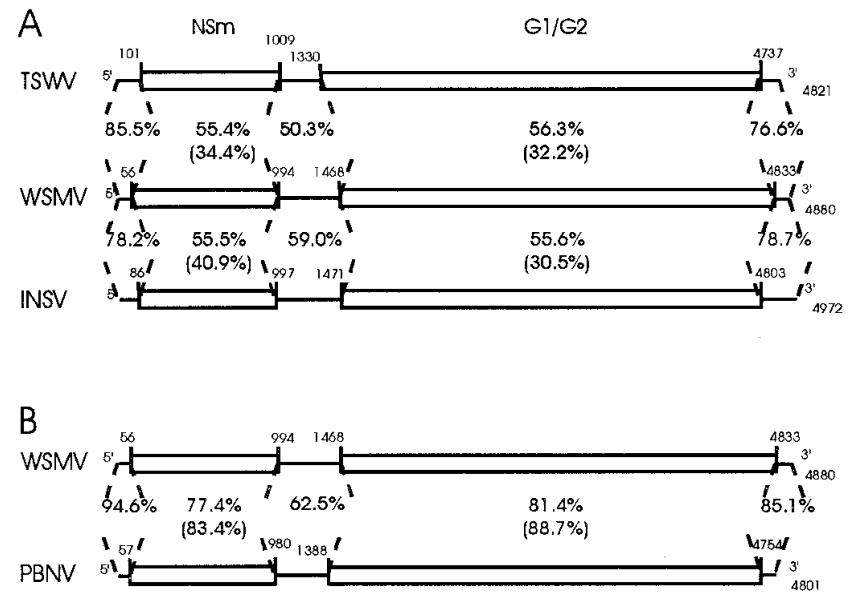

Fig. 4. A, Comparison of the genomic M RNA molecule of watermelon silver mottle virus (WSMV) with those of tomato spotted wilt virus (TSWV) (serogroup I) and impatiens necrotic spot virus (INSV) (serogroup III). B, Comparison of the genomic M RNA molecule of WSMV with that of peanut bud necrosis virus (PBNV) (serogroup IV). The positions of the genomic sequences are numbered from the $5^{\prime}$ ends of the viral RNAs. The percentages refer to nucleotide identity in both the noncoding and coding regions between the viruses listed. The amino acid identity of the M RNA-encoded nonstructural and G1/G2 proteins are in parentheses. The sequences used for comparison were from Kormelink et al. (18) for TSWV, Law et al. (25) for INSV, and Satyanarayana et al. (36) for PBNV. 
30.5 , and $88.7 \%$ with those of TSWV, INSV, and PBNV, respectively. The N-terminal regions (the first 85 amino acids) of the $\mathrm{G} 1 / \mathrm{G} 2$ proteins were hypervariable among the four viruses compared, and the central and C-terminal regions were more conserved.

Detection of mRNAs of the M RNA. The nucleotide sequence of WSMV M RNA predicted the presence of two ORFs in an ambisense organization. To detect mRNAs transcribed from both strands, total RNA extracted from WSMV-infected $N$. benthamiana plants was analyzed by Northern blotting using strand-specific riboprobes to parts of the two predicted ORFs. Both v and vc strands of the genomic 4.9-kb M RNA were detected (Fig. 5, lanes $2,4,6$, and 8), indicating that both negative- and positive-polarity M RNAs are present in infected cells. In addition to the M RNA species of genomic length, a v-sense RNA species of $1.0 \mathrm{~kb}$ (Fig. 5 , lane 4) was detected when the riboprobe NSm-vc corresponding to the NSm ORF was used for hybridization. In addition, the riboprobe G1/G2-v, corresponding to the ORF of the glycoprotein precursor, hybridized with a RNA species of $3.4 \mathrm{~kb}$ (Fig. 5, lane 6). The 1.0- and 3.4-kb RNA species were not found when probes NSm-v and G1/G2-vc were used for hybridization (Fig. 5, lanes 2 and 8). The 1.0-, 3.4-, and 4.9-kb RNAs were not detected in the total RNA extracted from the healthy controls (data not shown).

Viral RNAs extracted from cesium sulfate-purified nucleocapsids were analyzed by Northern blotting with the strand-specific riboprobes. The v- and vc-sense RNAs of genomic length were also detected (Fig. 5, lanes 1, 3, 5, and 7), indicating that both positive and negative polarity of the M RNA are encapsidated. Distinct RNA species of $1.0 \mathrm{~kb}$ (Fig. 5, lane 3) and $3.4 \mathrm{~kb}$ (Fig. 5, lane 5) were also detected with the NSm-vc and G1/G2-v probes, respectively. The amounts of these RNAs were nearly equal to the amount of genomic M RNA as judged from the intensity of hybridization reaction. Such RNA species were not detected when the NSm-v and G1/G2-vc probes (Fig. 5, lanes 1 and 7, respectively) were used to hybridize the RNA prepared from the purified nucleocapsids. The hybridization pattern of total RNA prepared from WSMV-infected tissue was similar to that of viral RNAs prepared from nucleocapsids (Fig. 5, lanes 2, 4, 6, and 8). In addition to the major 4.9- and 1.0-kb RNA species, some additional minor species of 2.8 to $3.0 \mathrm{~kb}$ associated with a smear background were also noticed when the NSm-vc probe was used (Fig. 5, lane 4). These minor RNA species and the background were considered the premature intermediates of the v-sense S RNA during replication or the degraded forms of the $\mathrm{v}$-sense $\mathrm{S}$ RNA during preparation. When total RNA extracted from the healthy plants was used for hybridi- zation with any of the four probes, no radioactive signals were observed (data not shown).

In view of the location and polarity of the probes on the physical map of the M RNA, and in view of the size of the NSm ORF and the glycoprotein precursor ORF encoded by this RNA segment, the 1.0-kb RNA and the 3.4-kb RNA were considered as the messages of the NSm gene and the G1/G2 gene transcribed from the vc and $\mathrm{v}$ strands of the M RNA, respectively. These results substantiate the ambisense coding strategy of the WSMV M RNA.

\section{DISCUSSION}

Although different methods $(6,23,39)$ were tried to purify virions of WSMV, we failed to obtain a sufficient amount for the extraction of the genomic ssRNAs to construct a cDNA library. In this study, the ds form of the M RNA extracted from WSMV-infected $C$. quinoa was successfully used for cDNA cloning. This method has also been applied previously for sequencing the complete WSMV S RNA $(42,44)$. The local lesion host $C$. quinoa inoculated with a high dosage of the virus usually produced numerous coalescent lesions 4 to 5 days after inoculation. The dsRNAs of the three tospoviruses extracted from $C$. quinoa were in larger quantity and much cleaner without smeared background after electrophoresis as compared with those from $N$. benthamiana (data not shown). In addition, the dsRNAs extracted from $C$. quinoa contained a greater amount of the L RNA that was absent or hardly detectable when the dsRNAs were extracted from $N$. benthamiana (data not shown). We feel that the extraction of the dsRNAs of tospoviruses from $C$. quinoa is an easier process than the extraction of viral ssRNAs from purified virions and appears to be a good alternative approach for cDNA cloning.

A consensus 3'-terminal nucleotide sequence of at least eight nucleotides has been found in the $\mathrm{L}, \mathrm{M}$, and $\mathrm{S}$ genomic segments of virus species within each genus of the family Bunyaviridae (9). The terminal sequences may contain important regulatory signals, such as recognition sites for the viral polymerase in genome transcription and replication (4). The consensus sequences of eight nucleotides at the $5^{\prime}$ and $3^{\prime}$ ends of the $M$ and S RNAs of WSMV, TSWV, INSV, and PBNV indicate that they all belong to the same genus Tospovirus of the family Bunyaviridae. The complete nucleotide sequence of the M RNA of WSMV encodes a NSm protein in the v strand and the precursor to the G1 and G2 glycoproteins in the vc strand. This ambisense arrangement of the M RNA of WSMV was evidenced from Northern blot analysis, which re-

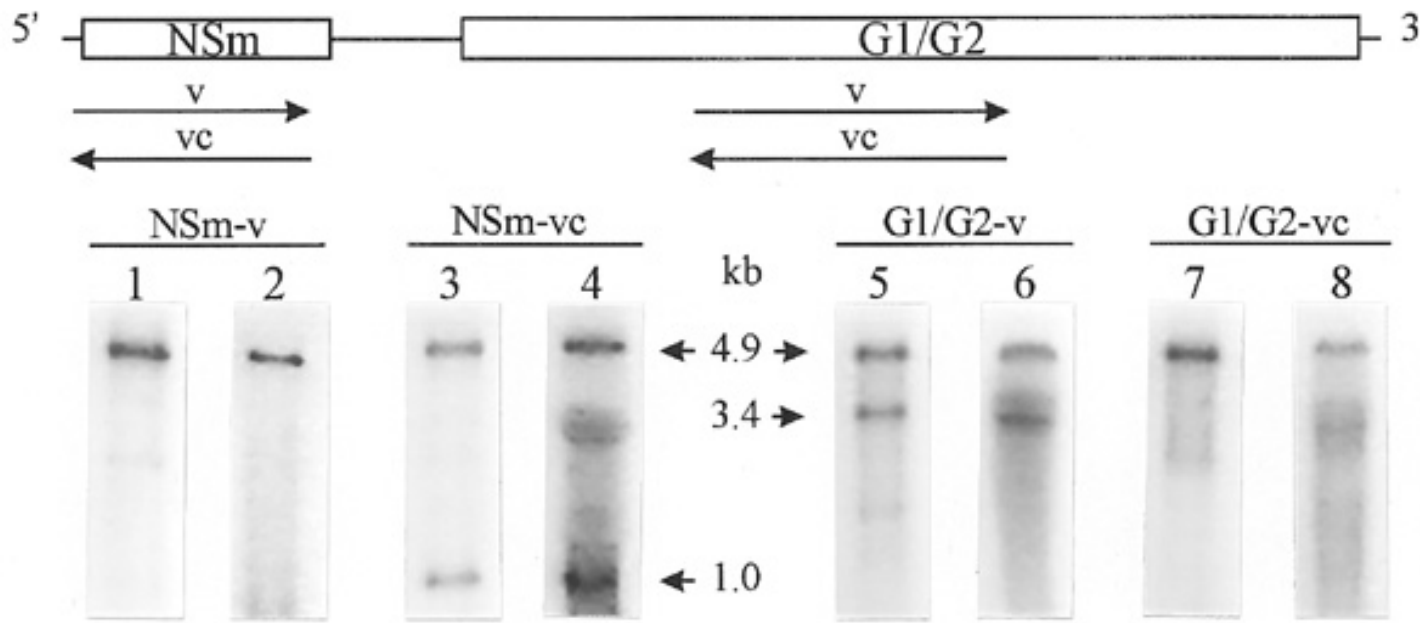

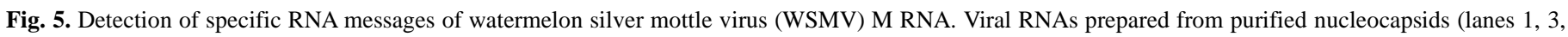

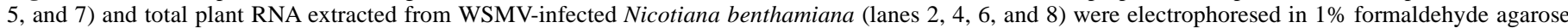

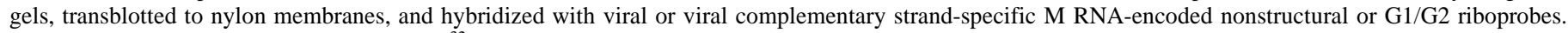

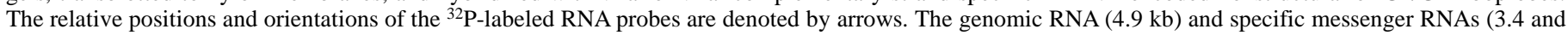
$1.0 \mathrm{~kb}$ ) detected are marked. 
vealed the presence of a v-sense 1.0-kb and a vc-sense 3.4-kb messenger RNA in infected plant cells. An ambisense genomic organization is a characteristic of the S RNAs in the genera Phlebovirus and Tospovirus of the family Bunyaviridae (8). However, the presence of ambisense $\mathrm{M}$ and S RNAs is unique to the Tospovirus genus (10). Comparison of the complete nucleotide sequence of WSMV M RNA with the other tospoviruses, TSWV and INSV, solidifies the taxonomic position of WSMV as a member of the genus Tospovirus in the family Bunyaviridae.

A search for the NSm protein of WSMV in the EMBL protein database did not reveal significant homology with other known proteins, except for the NSm proteins of other tospoviruses (TSWV, INSV, and PBNV). Computer alignments of the glycoprotein precursor of WSMV with those of other animal-infecting counterparts in the family Bunyaviridae revealed 7.5 to $11.4 \%$ amino acid similarity with Germiston virus, Bunyamwera virus, and La Crosse virus in the genus Bunyavirus. Alignments of the sequences of glycoprotein precursors of TSWV and INSV with these bunyaviruses revealed 5.9 to $10.0 \%$ and 5.5 to $9.5 \%$ amino acid similarity, respectively. No significant homology to the other members of the family Bunyaviridae was found. There was a conserved motif, TSxWGCEExGLCAxxxG (amino acid positions 696 to 712 from the N-terminus of the WSMV glycoprotein precursor), in glycoproteins common to these members of the genera Bunyavirus and Tospovirus. These results indicate that the glycoprotein precursors of tospoviruses are distantly related to those of viruses in the genus Bunyavirus.

Comparison of the amino acid sequences of the NSs and $\mathrm{N}$ genes of WSMV with those of TSWV and INSV revealed that their percentages of identity are fairly low, 12.1 to $13.7 \%$ for the NSs gene and 30.2 to $32.9 \%$ for the $N$ gene $(14,42,44)$. In this study, comparison at the amino acid level of the NSm and G1/G2 genes of WSMV with those of INSV and TSWV revealed that their percentages of identity were only 34.4 to $40.9 \%$ for the NSm gene and 30.5 to $32.2 \%$ for the G1/G2 gene. These results further strengthen the suggestion that WSMV has a phylogenetically distant relationship with TSWV (serogroup I) and INSV (serogroup III).

Although the N proteins of WSMV and PBNV have strong crossreactions serologically, WSMV induces severe systemic symptoms in most cucurbit species and does not infect peanut, while PBNV causes local infection in cucurbits $(31,42)$. To become a distinct species of the Tospovirus genus, it was agreed in a recent symposium on tospoviruses that the $\mathrm{N}$-protein sequence should show less than $90 \%$ amino acid sequence identity with that of any other tospovirus species that has been described (12). The N protein of WSMV shares $86 \%$ amino acid identity with that of PBNV $(35,42)$, which is below the taxonomic threshold level. This, coupled with the fact that the NSs protein of WSMV shares $83 \%$ amino acid identity with that of PBNV (35), suggests that they are two distinct species in serogroup IV.

Recently, the M RNA of PBNV (serogroup IV) has been determined as 4,801 nucleotides in length (36), slightly shorter than that of WSMV (4,880 nucleotides). In this study, the overall nucleotide identity of the M RNA (79.6\%) and the amino acid identities of the NSm and $\mathrm{G} 1 / \mathrm{G} 2$ proteins (83 and $88 \%$, respectively) of WSMV compared with those of PBNV further support that they are distinct tospoviruses. Moreover, the comparisons of the reported gene products of WSMV and PBNV indicated that the structural $\mathrm{N}$ and G1/G2 proteins are more closely related and the nonstructural NSs and NSm proteins are much more variable. The similarity and variability of the M and S RNAs, coupled with the differences in host reactions, indicate that WSMV and PBNV are two related and yet distinct species in serogroup IV of tospoviruses.

The $5^{\prime}$ and $3^{\prime}$ termini of the M RNA of WSMV were mostly complementary, perhaps resulting in the formation of a stable panhandle structure. Panhandle structures are believed to be important in the formation of pseudocircular RNA structures in other viruses of the family Bunyaviridae. These panhandle structures are thought to be important for encapsidation of the full-length $\mathrm{v}$ and $\mathrm{vc}$ strands of viral RNAs by the $\mathrm{N}$ protein for packaging into virions (8). Both full-length $\mathrm{v}$ and $\mathrm{vc}$ strands of the M RNA were detected in purified nucleocapsid preparations of WSMV, indicating that both the negative and positive polarity of M RNA are encapsidated. This is similar to the previous investigations on TSWV (18) and INSV (25).

In this investigation, the 1.0- and 3.4-kb RNAs, which may be messages for the NSm and G1/G2 genes, respectively, were also found to be associated with purified nucleocapsids. It was not proved that the nucleocapsids with the putative messages were released from the enveloped particles or were originally free in the cytoplasm of plant cells. We don't consider these two RNAs as defective RNAs, since their corresponding complementary strands were not found. It may be argued that the 1.0- and 3.4-kb RNAs were just associated with the nucleocapsids, but not truly encapsidated. However, the unprotected RNAs would probably not remain intact after the harsh procedure of nucleocapsid purification. Also, if the RNAs were not encapsidated, they would form a complex with a different density from the purified nucleocapsids and would sediment to a different position after cesium sulfate centrifugation. Alternatively, the two putative messenger RNAs could bind with other viral proteins, such as the NSm protein or other host proteins to form a ribonucleoprotein complex that could copurify with nucleocapsids during cesium sulfate isopycnic centrifugation. However, the nucleocapsids purified in this investigation contained a major protein of $31 \mathrm{kDa}$ that reacted with antiserum to the $\mathrm{N}$ protein of WSMV (data not shown). This fact, coupled with the large quantity of the 1.0- and 3.4-kb RNAs detected, does not support their binding with other viral or host proteins. Thus, our results suggest that the putative messages are possibly also encapsidated. These results have some resemblance to the phenomenon found by Raju and Kolakofsky (29), who reported that a minor amount of mRNA of La Crosse bunyavirus S RNA found in late infection was encapsidated. The authors concluded that the nucleocapsid recognition sequence is present at the $5^{\prime}$ end of the $\mathrm{v}$ and $\mathrm{vc}$ genomic RNAs, and the presence of the host primer at the $5^{\prime}$ end of the mRNA does not confer immunity to encapsidation. Thus, we speculate that the mRNAs for NSm and G1/G2 genes may still contain the correct context at the $5^{\prime}$ end for encapsidation with the $\mathrm{N}$ protein. The significance of this finding remains to be further investigated.

\section{ACKNOWLEDGMENTS}

This study was supported by grant NSC 84-2321-B005-071 from the National Science Council of the Republic of China on Taiwan. The GenBank accession number of the nucleotide sequence reported in this paper is U75379.

\section{LITERATURE CITED}

1. Adam, G., Yeh, S. D., Reddy, D. V. R., and Green, S. K. 1993. Serological comparison of tospovirus isolates from Taiwan and India with impatiens necrotic spot virus and serogroups of tomato spotted wilt virus. Arch. Virol. 130:237-250.

2. Bailey, J. M., and Davidson, N. 1976. Methylmercury as a reversible denaturing agent for agarose gel electrophoresis. Anal. Biochem. 70:75-85.

3. Bairoch, A. 1991. PROSITE: A dictionary of sites and patterns in proteins. Nucleic Acids Res. 19:2241-2245.

4. de Haan, P., de Avila, A. C., Kormelink, R., Westerbroek, A., Gielen, J. J. L., Peters, D., and Goldbach, R. W. 1992. The nucleotide sequence of the S RNA of impatiens necrotic spot virus, a novel tospovirus. FEBS (Fed. Eur. Biochem. Soc.) Lett. 306:27-32.

5. de Haan, P., Kormelink, R., de Oliveira Resende, R., van Poelwijk, F., Peters, D., and Goldbach, R. 1991. Tomato spotted wilt virus L RNA encodes a putative RNA polymerase. J. Gen. Virol. 71:2207-2216.

6. de Haan, P., Wagemarker, L., Peters, D., and Goldbach, R. 1989. Molecular cloning and terminal sequence determination of the $S$ and $M$ RNAs of tomato spotted wilt virus. J. Gen. Virol. 70:3469-3473.

7. de Haan, P., Wagemarker, L., Peters, D., and Goldbach, R. 1990. The S RNA segment of tomato spotted wilt virus has an ambisense character. J. 
Gen. Virol. 71:1001-1007

8. Elliot, R. M. 1990. Molecular biology of the Bunyaviridae. J. Gen. Virol. 71:501-522.

9. Francki, R. I. B., Fauquet, C. M., Knudson, D. L., and Brown, F. 1991. Classification and nomenclature of viruses. Fifth report of the International Committee on Taxonomy of Viruses. Arch. Virol. Suppl. 2:281-283.

10. German, T. L., Ullman, D. E., and Moyer, J. W. 1992. Tospoviruses: Diagnosis, molecular biology, phylogeny, and vector relationships. Annu. Rev. Phytopathol. 30:315-348.

11. Ghanekar, A. M., Reddy, D. V. R., Iizuka, N., Amin, P. W., and Gibbson, R. W. 1979. Bud necrosis of groundnut (Arachis hypogaea) in India caused by tomato spotted wilt virus. Ann. Appl. Biol. 93:173-179.

12. Goldbach, R., and Kuo, G. 1996. Introduction. The proceedings of the International Symposium on Tospovirus and Thrips of Floral and Vegetable Crops. Acta Hortic. 431:21-26.

13. Gubler, U., and Hoffman, B. J. 1983. A simple and very efficient method for generating cDNA libraries. Gene 25:263-269.

14. Heinze, C., Maiss, E., Adam, G., and Casper, R. 1995. The complete nucleotide sequence of the S RNA of a new Tospovirus species, representing serogroup IV. Phytopathology 85:683-690.

15. Henikoff, S. 1984. Unidirectional digestion with exonucleoase III creates targeted breakpoints for DNA sequencing. Gene 28:351-359.

16. Holmes, D. S., and Quigley, M. 1981. A rapid boiling method for the preparation of bacterial plasmids. Anal. Biochem. 114:193-197.

17. Iwaki, M., Honda, Y., Hanada, K., Tochihara, H., Yonaha, T., Hokama, K., and Yokoyama, T. 1984. Silver mottle disease of watermelon caused by tomato spotted wilt virus. Plant Dis. 68:1006-1008.

18. Kormelink, R., de Haan, P., Meurs, C., Peters, D., and Goldbach, R. 1992. The nucleotide sequence of the M RNA segment of tomato spotted wilt virus, a bunyavirus with two ambisense RNA segments. J. Gen. Virol. 73:2795-2804

19. Kormelink, R., de Haan, P., Peters, D., and Goldbach, R. 1992. Viral RNA synthesis in tomato spotted wilt virus-infected Nicotiana rustica plants. J. Gen. Virol. 73:687-693.

20. Kormelink, R., Kitajima, E. W., de Haan, P., Zuidema, D., Peters, D., and Goldbach, R. 1991. The nonstructural protein (NSs) encoded by the ambisense S RNA segment of tomato spotted wilt virus is associated with fibrous structures in infected plant cells. Virology 181:459-468.

21. Kormelink, R., Storms, M., Van Lent, J., Peters, D., and Goldbach, R. 1994. Expression and subcellular location of the NSm protein of tomato spotted wilt virus (TSWV), a putative viral movement protein. Virology 200:56-65

22. Kyte, J., and Doolittle, R. F. 1982. A simple method for displaying the hydropathic character of a protein. J. Mol. Biol. 157:105-132.

23. Law, M. D., and Moyer, J. W. 1990. A tomato spotted wilt-like virus with a serologically distinct N protein. J. Gen. Virol. 71:933-938.

24. Law, M. D., Speck, J., and Moyer, J. W. 1991. Nucleotide sequence of the $3^{\prime}$ non-coding region and $\mathrm{N}$ gene of the S RNA of a serologically distinct tospovirus. J. Gen. Virol. 72:2597-2601.

25. Law, M. D., Speck, J., and Moyer, J. W. 1992. The M RNA of impatiens necrotic Tospovirus (Bunyaviridae) has an ambisense genomic organization. Virology 188:732-741.

26. Maniatis, T., Fritsch, E. F., and Sambrook, J. 1982. Molecular Cloning: A Laboratory Manual. Cold Springs Harbor Laboratory, Cold Springs Harbor, NY.

27. Mohamed, N. A. 1981. Isolation and characterization of subviral struc- tures from tomato spotted wilt virus. J. Gen. Virol. 53:197-206.

28. Mohamed, N. A., Randles, J. W., and Francki, R. I. B. 1973. Protein composition of tomato spotted wilt virus. Virology 56:12-21.

29. Raju, R., and Kolakofsky, D. 1987. Unusual transcripts in La Crosse virus-infected cells and the site for nucleocapsid assembly. J. Virol. 61: 667-672.

30. Rao, J. K. M., and Agros, P. 1986. A conformational preference/parameter to predict helices in integral membrane proteins. Biochem. Biophys. Acta 869:197-214.

31. Reddy, D. V. R., Ratna, A. S., Sudarshana, M. R., Poul, F., and Kumar, I. K. 1992. Serological relationships and purification of bud necrosis virus, a tospovirus occurring in peanut (Arachis hypogaea L.) in India. Ann. Appl. Biol. 120:279-286.

32. Ruoslahti, E., and Pierschbacher, M. D. 1986. Arg-Gly-Asp: A versatile cell recognition signal. Cell 44:517-518.

33. Sambrook, J., Fritsch, E. F., and Maniatis, T. 1989. Molecular Cloning: A Laboratory Manual, 2nd ed. Cold Springs Harbor Laboratory, Cold Springs Harbor, NY

34. Sanger, F., Nicklen, S., and Coulson, A. R. 1977. DNA sequencing with chain-terminating inhibitors. Proc. Natl. Acad. Sci. U.S.A. 83: 571-579.

35. Satyanarayana, T., Mitchell, S. E., Reddy, D. V. R., Brown, S., Kresovich, S., Jarret, R., Naidu, R. A., and Demski, J. W. 1996. Peanut bud necrosis tospovirus S RNA: Complete nucleotide sequence, genome organization and homology to other tospoviruses. Arch. Virol. 141:85-98.

36. Satyanarayana, T., Mitchell, S. E., Reddy, D. V. R., Kresovich, S., Jarret, R., Naidu, R. A., Gowda, S., and Demski, J. W. 1996. The complete nucleotide sequence and genome organization of the M RNA segment of peanut bud necrosis tospovirus and comparison with other tospoviruses. J. Gen. Virol. 77:2347-2352.

37. Schwinghamer, M. W., and Symons, R. H. 1977. Translation of the four major RNA species of cucumber mosaic virus in plant and animal cell free systems and in toad oocytes. Virology 79:88-108.

38. Storms, M. M. H., Kormelink, R., Peter, D., Van Lent, J. W. M., and Goldbach, R. W. 1995. The nonstructural NSm protein of tomato spotted wilt virus induces tubular structures in plant and insect cells. Virology 214:485-493.

39. Tas, P. W. L., Boerjan, M. L., and Peters, D. 1977. The structural proteins of tomato spotted virus. J. Gen. Virol. 36:267-279.

40. Van Den Hurk, J., Tas, P. W. L., and Peters, D. 1977. The ribonucleic acid of tomato spotted wilt virus. J. Gen. Virol. 36:81-91.

41. Verwoerd, T. C., Dekker, B. M. M., and Hoekema, A. 1989. A smallscale procedure for the rapid isolation of plant RNAs. Nucleic Acids Res. 17:2362.

42. Yeh, S.-D., and Chang, T.-F. 1995. Nucleotide sequence of the $\mathrm{N}$ gene of watermelon silver mottle virus, a proposed new member of the genus $T o$ spovirus. Phytopathology 85:58-64.

43. Yeh, S.-D., Lin, Y.-C., Cheng, Y.-H., Jih, C.-L., Chen, M.-J., and Chen, C.-C. 1992. Identification of tomato spotted wilt-like virus infecting watermelon in Taiwan. Plant Dis. 76:835-840.

44. Yeh, S. D., Sun, I. J., Ho, H. M., and Chang, T. F. 1996. Molecular cloning and nucleotide sequence analysis of the S RNA of watermelon silver mottle tospovirus. Acta Hortic. 431:244-260.

45. Zuker, M., and Stiegler, P. 1981. Optimal computer folding of large RNA sequences using thermodynamics and auxiliary information. Nucleic Acids Res. 9:133-148. 